\title{
Urinary dipsticks: efficacy as predictor of urinary tract infections
}

\begin{abstract}
Urinalysis and culture are the two most common investigations performed in a microbiology laboratory. Commercial Dipsticks which detect proteins and various enzymes may offer a rapid and inexpensive alternative to urinalysis and culture. An evaluation of the utility of urinary dipsticks (Multistix $10 \mathrm{SG}$ ) in predicting urinary tract infections (UTI) was undertaken. A total of 667 urine samples were evaluated from May to August 2012. Sensitivity, specificity, negative and positive predictive values were evaluated. The sensitivity for predicting UTI was low: $62 \%$ for leukocyte esterase and nitrite; $67 \%$ for leukocyte esterase and proteinuria while it was $74 \%$ for leukocyte esterase, nitrite and proteinuria, all three together. However, the negative predictive value (NPV) for UTI was better. NPV for UTI with leukocyte esterase and nitrite was $90.3 \%$; for leukocyte esterase and proteinuria was $90.6 \%$ while it was 91.5 $\%$ for leukocyte esterase, nitrite and proteinuria together. The positive predictive value was very low. Urinary dipsticks therefore appear to be better tools for excluding UTIs and not for diagnosing UTI.
\end{abstract}

Keywords: urinalysis, urine culture, urinary tract infections, urinary dipsticks
Volume 2 Issue I - 2016

\author{
Ruchika Bagga,Alka Girotra \\ Department of Microbiology, Fortis Healthcare, India
}

Correspondence: Ruchika Bagga, Department of Microbiology, Fortis Healthcare, Gurgaon, Haryana, India, Email ruchikaa.bagga@gmail.com

Received: January 19, 2015 | Published: March 30, 2016

\section{Introduction}

Urinalysis and urine culture are the commonest tests ordered by clinicians. These tests account for almost $60-70 \%$ of the workload of any clinical microbiology lab. Some large labs get as much as 200300 urine samples per day. Given that majority of them (around 80\%) would eventually show no growth on culture, there is huge interest in ways to screen out these negative samples. Empiric treatment of urinary tract infection is one of the commonest causes of inappropriate and unnecessary antibiotic usage. ${ }^{1}$ In judicious antibiotic usage is most common factor leading to multi drug resistant organisms almost $20 \%$ of laboratory samples have antibiotic resistance.,3 Dipstick provide a less time consuming and inexpensive alternative and is most commonly used for near patient/point of care testing by clinicians in primary care ${ }^{4-7}$ Reliable screening tests/near patients tests (NPTs)/ point of care tests for diagnosis of UTI would facilitate early diagnosis and treatment and if negative would help avoid unnecessary, expensive time consuming culture.

Of late, there has been a lot of interest in the urine screening tests and these have been a topic of research in recent times.

Many studies have been done on the comparison of various dipstick parameters (widely used as screening tests) with bacterial culture. The reports however, have been conflicting. The present study was undertaken to evaluate the utility of various parameters assessed by use of urinary dipsticks in predicting urinary tract infections.

\section{Materials and methods}

An observational study was carried out in Department of Microbiology of a tertiary care hospital between May to August 2012 to evaluate the efficacy of urinary dipsticks in predicting UTI. A total of 667 urine samples were analysed.

The sample size calculation was done by taking into account the total number of urine samples received (20000 samples/year) Using the data for sensitivity and specificity $(-70 \%)$ available from literature and assuming the type 1 error to be $0.5 \%$ Multistix $10 \mathrm{SG}$ (Seimens) reagent strips were used in the study. Calibrated loops were used to culture $1 \mathrm{~mL}$ of urine on to MacConkey and Sheep Blood agar plates (Biomerieux). The criterion for clinically significant bacteriuria was a pure/predominant culture of $10^{\wedge} 5 \mathrm{cfu} / \mathrm{ml}$ of the microorganism.

The nitrite test depends upon the presence of nitrites in urine. The bacteria in urine produce an enzyme nitrate reductase which reduces nitrate to nitrite. So if bacteria are present in urine the nitrite test will be positive.

The leukocyte esterase test uses derivatized pyrrole amino acid ester to diagnose leukocyte esterase in WBCs.

Comparison of leukocyte esterase, nitrite and presence of proteins in urine with culture was done.

Sensitivity, specificity, positive predictive value and negative predictive values of each of these markers was calculated.

Table I Sensitivity (in comparison to gold standard i.e. culture)

\begin{tabular}{lc}
\hline Leukocyte Esterase & 51.2 \\
Nitrite & 37.8 \\
Proteinuria & 44.1 \\
Leukocyte Esterase+Nitrite & 62.2 \\
Leukocyte Esterase+Proteinuria & 67.7 \\
Nitrite+Proteinuria & 64.6 \\
Leukocyte Esterase+Nitrite+Proteinuria & 74 \\
\hline
\end{tabular}


Table 2 Specificity

\begin{tabular}{ll}
\hline Leukocyte Esterase & 92 \\
Nitrite & 88.1 \\
Proteinuria & 78 \\
Leukocyte Esterase+Nitrite & 82.8 \\
Leukocyte Esterase+Proteinuria & 73 \\
Nitrite+Proteinuria & 30.7 \\
Leukocyte Esterase+Nitrite+Proteinuria & 65.7 \\
\hline
\end{tabular}

Table 3 Positive predictive value

\begin{tabular}{ll}
\hline Leukocyte Esterase & 60.2 \\
Nitrite & 42.9 \\
Proteinuria & 32 \\
Leukocyte Esterase+Nitrite & 45.9 \\
Leukocyte Esterase+Proteinuria & 37.1 \\
Nitrite+Proteinuria & 18 \\
Leukocyte Esterase+Nitrite+Proteinuria & 33.7 \\
\hline
\end{tabular}

Table 4 Negative predictive value

\begin{tabular}{ll}
\hline Leukocyte Esterase & 88.9 \\
Nitrite & 85.8 \\
Proteinuria & 85.6 \\
Leukocyte Esterase+Nitrite & 90.3 \\
Leukocyte Esterase+Proteinuria & 90.6 \\
Nitrite+Proteinuria & 78.7 \\
Leukocyte Esterase+Nitrite+Proteinuria & 91.5 \\
\hline
\end{tabular}

\section{Results}

Total cultures processed: 667 urine samples.

Samples which were culture positive: 127.

\section{Discussion}

Use of dipsticks/NPTs/Point of care tests definitely decreases the time and the cost of testing. Application of such a screening test to exclude UTI would save both resources and time.

The essential requirements of a screening test for UTI are high sensitivity so that no case of UTI is missed and high specificity so that no case of UTI is falsely diagnosed as such. There should be minimal false negatives and false positives.

The predictive value of a positive/negative test should therefore be close to $100 \%$ so that the question that whether the patient has/doesn't have UTI is reliably answered.

The leukocyte esterase test in our study was found to be non sensitive indicator for UTI. Positive predictive value of this test was found to be low $(60.2 \%)$ but a negative leukocyte esterase test $(89 \%)$ excluded UTI indicating good NPV.

The reasons for low sensitivity are reported in literature. False positive esterase test can be due to lysis of WBCs with release of esterase and in such cases microscopy of urine for WBC will be negative. ${ }^{8}$ Also sterile (bacterial) pyuria is known to occur in a large number of cases. Interference in this test result may also be on account of contamination with vaginal secretions in case of girls.

The above factors contribute to Leukocyte esterase alone being not a very sensitive indicator $(51.2 \%)$ for prediction of UTI but as the test has high NPV, the negative result is a good test for excluding UTI. Similar findings have been reported by Hulburt et al., ${ }^{9}$ and Wenk et al. ${ }^{10}$ where a negative leukocyte esterase was shown to be a better predictor of absence of Urinary tract infections compared to presence of nitrite.

The nitrite test was found to have a relatively low sensitivity $(37.8 \%)$ and somewhat high specificity $(88.1 \%)$. However, this parameter has its own shortcomings. Nitrite detection in urine is usually a result of presence of nitrate reductase in bacteria like E.coli, Proteus, Klebsiella, Citrobacter and Enterobacter which reduce nitrate to nitrite. ${ }^{11-13}$ Some UTI cases caused by bacteria like Streptococci cannot be detected as Streptococci do not produce the enzyme nitrate reductase. ${ }^{14}$

The nitrate to nitrite conversion takes several hours. ${ }^{15}$ Use of overnight first morning samples increases the sensitivity of nitrite test. Inadequate nitrate intake in diet (beans, spinach being good sources) and presence of interference e.g. like by presence of antimicrobials in urine would all lead to false negative results.

Delay in screening/plating could lead to false positive results as most common urinary pathogen (e.g E.coli) multiplies within 20minutes and this could lead to false positive findings in case a few Esch coli fall into urine during collection.

Combining nitrite parameter with leukocyte esterase, the sensitivity for prediction of UTI increased $(\sim 63 \%)$ but the specificity fell $(\sim 83 \%)$ in our study. ${ }^{16-19}$ The negative predictive value for UTI using the two parameters was high $(90.6 \%)$. Thus the two tests together increased overall accuracy for prediction of UTI.

This is in concordance with the results reported by several investigators on the efficacy of these two parameters together to predict UTI. It was concluded that the nitrite and leukocyte esterase together have higher negative predictive value. ${ }^{20-22}$

Proteinuria, though not a very specific test $(78 \%)$ by itself, but along with above two parameters helped improve the overall negative predictive value of dipstick tests $(92 \%)$ in excluding UTI. This is because with inclusion of proteinuria, patients with glomerular disease also get included.

However, the positive predictive value of these three tests combined was only $34 \%$. Inclusion of symptomatic patients may increase the sensitivity of these tests. So, the dipstick method testing for all the three parameters may be of value in excluding UTI as point of care test in the physicians office. ${ }^{9,23}$

\section{Conclusion}

The urinalysis thus is not a sufficiently strong predictor of UTI to be relied on as a sole test. Positive predictive value is too low to predict UTI. A urine cultures thus remain the gold standard for diagnosis of UTI. 
Given the low positive predictive value of leukocyte esterase, nitrite and proteinuria in the present study, use of these tests alone would result in unnecessary antibiotics being prescribed to one third to one half of the patients. Culture result is therefore necessary so that the correct treatment is given to the correct patient. The negative predictive value is good enough to rule out UTI. It could thus help save on valuable time and resources in excluding UTI. They however, carry a lot of value in excluding urinary tract infections since they have a very good negative predictive value. Performance of these tests in the physicians room/Emergency departments would also help in curbing unnecessary usage of antibiotics for empiric treatment of urinary tract infections. Also, it would have a tremendous impact in decreasing work load and saving valuable time and resources.

\section{Acknowledgements}

None.

\section{Conflict of interest}

The author declares no conflict of interest.

\section{References}

1. Fenwick E, Briggs A, Hawke C. Management of urinary tract infection in general practice: a cost effectiveness analysis. $\mathrm{Br} J$ Gen Pract. 2000;50(457):635-639.

2. House of Lords. House of Lords Select Committee on Science and Technology: $7^{\text {th }}$ report. Occasional Report. London: House of Lords; 1998.

3. Kusumi RK, Grover PJ, Kunin CM. Rapid detection of pyuria by leukocyte esterase activity. JAMA. 1981;245(16):1653-1655.

4. Ditchburn RK, Ditchburn JS. A study of microscopical and chemical tests for the rapid diagnosis of urinary tract infections in general practice. $\mathrm{Br} \mathrm{J}$ Gen Pract. 1990;40(339):406-408.

5. Schulz H, McCaffey T, Keys TF, et al. Acute cystitis: a prospective study of laboratory tests and duration of therapy. Mayo Clin Proc. 1984;59(6):391397.

6. Winkens RA, Leffers P, Trienkens TA, et al. The validity of urine examination for urinary tract infections in daily practice. Fam Pract. 1995;12(3):290-293.

7. Standing Medical Advisory Committee. Standing Medical Advisory Committee (SMAC) report: The path of least resistances. Occasional report 1998. London: Depatment of Health; 1998.

8. Lenke RR, Van Dorsten JP. The efficiency of the nitrite and microscopic analysis in predicting urine culture results. Am J Obstet Gynecol. 1981;140(4):427-429.
9. Hurlbut TA, Littenburg B. The diagnostic accuracy of rapid dipstick tests to predict urinary tract infection. Am J Clin Pathol. 1991;96(5):582-588.

10. Wenk RE, Dutta D, Rudert J, et al. Sediment microscopy, nitriuria and leukocyte esteriuria as predictors of significant bacteriuria. J Clin Lab Automation. 1982;2:117-21.

11. Zaman Z, Borremanns A, Verhaegen J, et al. Disappointing dipstick screening for urinary tract infection in hospital inpatients. J Clin Pathol. 1998;51(6):471-472.

12. Loo SYT, Scottolini AG, Luangphinith S, et al. Urine screening strategy employing dipstick analysis and selective culture:an evaluation. Am J Clin Pathol. 1984;81(5):634-642.

13. Powell HR, McCredie DA, Ritchie MA. Urinary nitrite in symptomatic and a symptomatic urinary infection. Arch Dis Child. 1987;62(2):138-140.

14. Zainal D, Baba A. The value of positive nitrites in screening asymptomatic bacteriuria amongst Malaysian school children. Southeast Asian J Trop Med Public Health. 1996;27(1):184-188.

15. Lachs MS, Nachamkin I, Edelstein $\mathrm{PH}$, et al. Spectrum bias in the evaluation of diagnostic tests: lessons from the rapid dipstick test for urinary tract infection. Ann Intern Med. 1992;117(2):135-140.

16. Carroll KC, Hale DC, VonBoerum DH, et al. Laboratory evaluation of urinary tract infections in an ambulatory clinic. Am J Clin Pathol. 1994;101(1):100-103.

17. Fowlis GA, Waters J, Williams G. The cost effectiveness of combined tests (Multistix) in screening for urinary tract infections. $J$ R Soc Med. 1994;87(11):681-682.

18. Nostrand JD, Junkins AD, Bartholdi RK. Poor predictive ability of urinalysis and microscopic examination to detect urinary tract infection. Am J Clin Pathol. 2000;113(5):709-713.

19. Propp DA, Weber D, Ciesla ML. Reliability of a urine dipstick in emergency department patients. Ann Emerg Med. 1989;18(5):560-563.

20. Shaw ST, Poon SY, Wong ET. Routine urinalysis. Is the dipstick enough? JAMA. 1985;253(11):1596-1600.

21. Semeniuk H, Church D. Evaluation of the leukocyte esterase and nitrite urine dipstick screening tests for detection of bacteriuria in women with suspected uncomplicated urinary tract infections. J Clin Microbiol. 1999;37(9):3051-3052.

22. Wiggelinkhuizen J, Maytham D, Hanslo DH. Dipstick screening for urinary tract infections. S Afr Med J. 1988;74(5):224-228.

23. Bailey BL. Urinanalysis predictive of urine culture results. J Fam Pract. $1995 ; 40(1): 45-50$ 